\title{
METAMAGNETIC STATES IN METALLIC NANOSTRUCTURES
}

\author{
V.S. Stepanyuk, ${ }^{a}$ W. Hergert, ${ }^{b}$ P. Rennert, ${ }^{b}$ K. Wildberger, ${ }^{c}$ R. Zeller ${ }^{c}$ and P.H. Dederichs ${ }^{c}$ \\ ${ }^{a}$ Max-Planck-Institut für Mikrostrukturphysik, Weinbergweg 2, D-06120 Halle, Germany \\ ${ }^{b}$ Fachbereich Physik, Martin-Luther-Universität, Fr.-Bach-Platz 6, D-06099 Halle, Germany \\ ${ }^{c}$ Institut für Festkörperforschung, Forschungszentrum Jülich, D-52425 Jülich, Germany
}

(Received 30 October 1996; accepted 19 November 1996 by H. Eschrig)

\begin{abstract}
Materials of mesoscopic dimensions can be produced by modern scanning microscopic and chemical techniques. This opens the possibility of creating new nanoscale magnets, which consist only of a few atoms. These systems may exhibit novel and unusual properties. We demonstrate, that metamagnetic states are possible in supported metallic nanostructures. This effect can lead to magnetic transitions between different magnetic states by a change of external parameters like temperature or applied fields. We apply the local approximation of the density functional theory and a recently developed KKR Green's function method to supported clusters. A detailed consideration of the $\mathrm{Cu}(001)$ surface with $3 d$ supported metallic nanostructures is presented. (C) 1997 Elsevier Science Ltd. All rights reserved
\end{abstract}

In the last years a rapid development of methods for the manipulation of single atoms and small clusters on metal surfaces was achieved. [1,2] Eigler et al. have shown, that the scanning tunneling microscope (STM) can be used to move atoms on a solid surface. For example they have built a chain of $4 \mathrm{Pt}$ atoms on a $\mathrm{Pt}(111)$ surface. [1] Röder et al. [2] have shown, that structures of various morphology can be simultaneously formed by diffusion controlled aggregation, which is controlled by the crystal face used and the temperature. Linear, two-dimensional or fractal aggregates can be grown in this way. Even the length of linear chains can be controlled by external parameters. Techniques which create artificial structures atom by atom may be limited by speed. The diffusion controlled aggregation gives structures of nearly the same number of atoms and the same shape with a high number density $\left(10^{11}-10^{14} \mathrm{~cm}^{-2}\right)$. Therefore, physical and chemical properties can be measured with conventional surface techniques. Using new techniques in molecular chemistry Gatteschi et al. [4] have demonstrated the possibility of creating molecular magnets like a ligated $\mathrm{Fe}_{10}$ cluster ("ferric wheel"). It was also reported recently that $\mathrm{Mn}_{12}$ clusters show magnetic bistability and that such property might be useful to produce new storage devices based on nanoscale magnets. [5] The novel nanostructures can show unusual magnetic properties, e.g. $4 d$ metals, which are nonmagnetic in the bulk, can be magnetic as small clusters on metal surfaces [6]. This theoretical prediction has been recently confirmed experimentally. [7]

We present results of first principle calculations which show, that small supported metallic nanostructures have a metamagnetic behaviour, which might lead to magnetic fluctuations between different magnetic states. This behaviour is connected to dynamical effects like quantum tunneling of magnetization (QTM) as discussed by Chudnovsky and Gunther. [8] To proof this effect, we discuss the results for metallic nanostructures of $3 d$ atoms on the $\mathrm{Cu}(001)$ surface. The results for different substrates and clusters of different geometries and sizes will be published elsewere. [9]

A KKR Green's function method for impurities and clusters at surfaces is used for the calculations. Details of the method can be found elsewhere. [10] The basic idea of the method is a hierarchical scheme for the construction of the Green's functions of the supported cluster by means of successive applications of Dyson's equations. The bulk Green's function is transformed to a Wannier-Bloch representation. The surface Green's function is calculated self-consistently from the bulk by replacing seven $\mathrm{Cu}$ layers by vacuum. After a transformation of the surface Green's function to a site rep- 
resentation the Dyson equation is used again to calculate self-consistently the Green's function of the supported cluster. Exchange and correlation effects are included in LSDA applying the potential of Vosko et al. [11] The full charge density is taken into account using a multipole expansion up to $l=6$. The potentials are assumed to be spherically symmetric inside the Wigner-Seitz spheres. In our procedure lattice relaxations are neglected. All atoms are fixed at the corresponding positions in the ideal crystal. This approximation will not seriously affect the results, because in most cases the relaxation energy is small in comparison to the corresponding spin polarization energy. [10] While large clusters might show non-collinear structure of the magnetic moments, such a situation is not likely for the clusters studied here.

The atoms of the clusters studied here occupy ideal lattice sites. We study dimers, trimers (linear chain) and tetramers (plain islands of 4 atoms). The nearest neighbour distance of the atoms in the cluster is the nearest neighbour distance of the $\mathrm{Cu}$ lattice. The dimer and the tetramer have only one non-equivalent site in the paramagnetic state, while the trimer has two non-equivalent sites (center atom (C) and edge atoms $\left.\left(E, E^{\prime}\right)\right)$. No competing magnetic exchange interactions occur as e.g. for a triangular structure, so that noncollinear states are unlikely.

Various magnetic states are possible for the trimer without destroying the chemical symmetry of the system. Ferromagnetic states (low spin ferromagnetic LSF, high spin ferromagnetic - HSF) have parallel moments at each site but the size might be different at the center and at the edge positions. The moments at the center and at the edge atoms are antiparallel in an antiferromagnetic (AF) state, but might be of different absolute value. In both cases, however, the two edge atoms (E,E') should have the same moment $\left(M_{E}=\right.$ $M_{E^{\prime}}$ ). A fourth state allowed by symmetry is an antisymmetric (AS) one. The magnetic moment at the central atom of the trimers is zero and the moments of the edge positions are antiparallel $\left(M_{E}=-M_{E^{\prime}}\right)$.

To our surprise we found the existence of these four magnetic configurations for practically all 3d-trimers on $\mathrm{Cu}(001)$. The absolute values of the magnetic moments at the center and edge atoms for the HSF and AF states as well as the moments of the edge atoms of the low spin (LS) and AS state differ only slightly. The total moments of the trimers for the HSF, LS, and the AF state are shown in Fig. 1.

Only $\mathrm{Ni}_{3}$ and $\mathrm{Ti}_{3}$ were found to be nonmagnetic. For the other cases very large moments occur. Despite this strong tendency to magnetism all four solutions exist in parallel as local minima of the total energy surface.

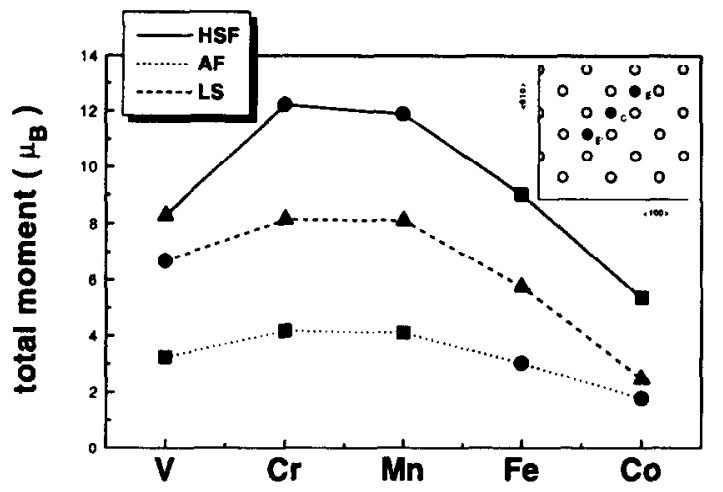

Fig. 1. Total moment for $3 d$ trimers on $\mathrm{Cu}(001)$ in the HSF, LSF, and AF state. The ground state configuration is marked by a filled square. The state next in energy is marked by filled circle. The state marked by the filled triangle has the highest energy.

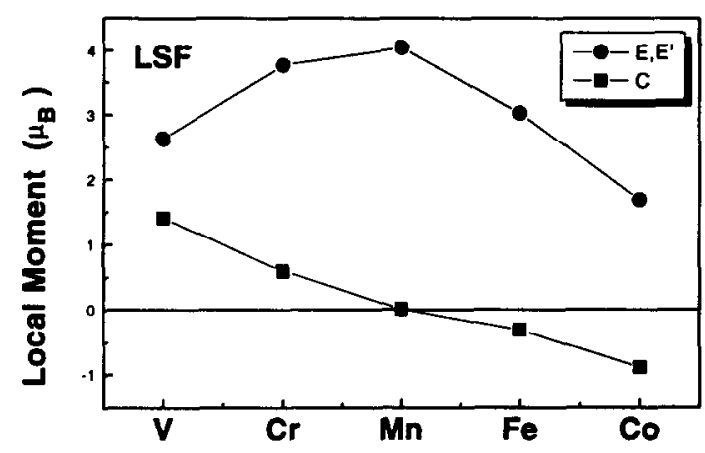

Fig. 2. Low spin state for $3 d$ trimers on $\mathrm{Cu}(001)$. The state changes its character from ferromagnetic to antiferomagnetic through the $3 d$ row.

The difference between total magnetic moments for the states investigated can be giant. We have obtained also the AS solutions for all magnetic trimers, but due to the fact that the total moment of the trimer in the AS state is zero and the higher energy compared to the AF, HSF and LS state, we will not discuss this state in detail here.

Total energy calculations are performed to find the ground states of the trimers. The energetic order of the states is also indicated in Figure 1. The ground state changes from $\mathrm{AF}$ for $\mathrm{V}, \mathrm{Cr}$ and $\mathrm{Mn}$ to $\mathrm{HSF}$ for $\mathrm{Fe}$ and Co. The low spin solution changes its character from ferromagnetic to antiferromagnetic as shown in Fig. 2.

In Fig. 3 the energy differences of the magnetic states with respect to the paramagnetic one are given for the LS, HSF and AF configuration. The energetic balance between the magnetic states is particularly delicate for V. Here the AF state has an energy only 8 meV/atom lower than the LSF state which itself has slightly lower energy than the HSF state. Another in- 


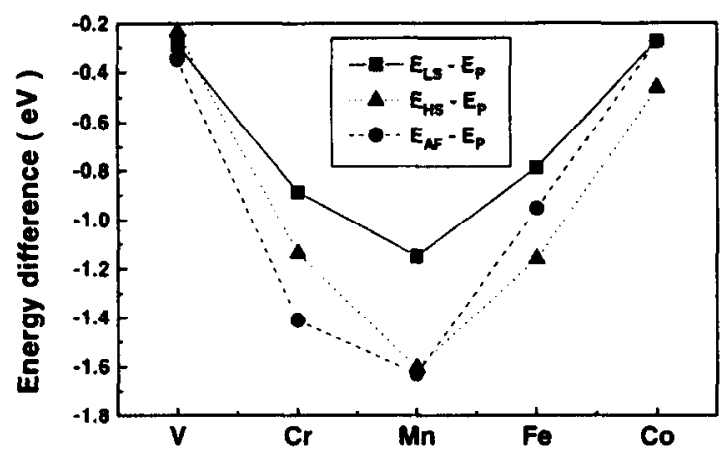

Fig. 3. Differences in total energy per atom between the various magnetic states and the paramagnetic one for $3 d$ trimers on $\mathrm{Cu}(001)$

Table 1. Local moments of the atoms (clockwise numbering of atoms) for the HS and LS ferromagnetic states and for the two antiferromagnetic states $A F_{1}$ and $\mathrm{AF}_{2}$ of the $\mathrm{V}_{4}$-tetramer (moments in units of $\mu_{B}$ )

\begin{tabular}{ccccc}
\hline state & $\mathbf{M}_{\mathbf{l}}$ & $\mathbf{M}_{2}$ & $\mathbf{M}_{3}$ & $\mathbf{M}_{4}$ \\
\hline HSF $(111)$ & 1.27 & 1.27 & 1.27 & 1.27 \\
LSF $(11 \uparrow 1)$ & 0.50 & 0.50 & 0.50 & 0.50 \\
AF $_{1}(111 !)$ & 1.90 & -1.90 & 1.90 & -1.90 \\
$\mathrm{AF}_{2}(1111)$ & 2.14 & 2.14 & -2.14 & -2.14 \\
\hline
\end{tabular}

teresting case is the Mn trimer, where the energy difference between the AF ground state and the HSF state is only $2 \mathrm{meV} /$ atom (corresponding to a temperature of $25 \mathrm{~K}$ ). Thus already small variations of external parameters like temperature or applied field could lead to transitions between these states, which in the case of $\mathrm{Mn}$ would change the total moment of the cluster by $7.8 \mu_{\mathrm{B}}$. Mn clusters on the $\mathrm{Cu}(001)$ surface can be considered as a canditate to study QTM effects. [8] Such large changes in the magnetic moments should be detectable in experiments.

It is well known [12], [13] that the moments and energies of free clusters show a nonmonotonic behaviour with cluster size. In line with this finding, previous calculations for $4 \mathrm{~d}$ nanostructures on $\mathrm{Ag}$ [6] showed a strong sensitivity of the moments on the size and structure of the supported clusters. To prove that metamagnetic behaviour is a common effect in metallic nanostructures, we have also performed calculations for the dimer and tetramer configurations. For the $V_{2}$ dimer we find both a ferromagnetic and an antiferromagnetic state with moments of 2.85 and $2.58 \mu_{B}$ respectively. The antiferromagnetic state has the lowest energy being about $0.2 \mathrm{eV} /$ atom lower than the ferromagnetic one.

Table 1 lists the moments of the tetramer. If we number the atoms of the tetramer clockwise, the following spin configurations are possible : $11 \uparrow 1,1 \uparrow 1$. , $1 \downarrow \uparrow 1$. Ferromagnetic coupling is obtained for a high spin and and a low spin state. As for the trimer the AF state with antiparallel coupling of nearest neighbours $111 \downarrow$ has the lowest energy being about 0.05 $\mathrm{eV}$ /per atom lower than for the HSF state, which itself has slightly energy lower than the two other solutions. Analogously to the different magnetic states discussed for the trimer, all four magnetic states of the tetramer do not destroy the chemical point symmetry $C_{4 v}$ of this system and thus do not lead to reconstruction.

At the end we will shortly discuss the reason for the large multiplicity of the magnetic states. The local moments for the HSF state and for the AF state of the trimer are very similar. The same is also true for the local moments of the ferromagnetic and AF states of the dimers, which agree very well with the moments of the single adatoms. Thus apparently the hybridization between the neighbouring adatoms is sufficiently weak that the magnetic interaction can be qualitatively described by a Heisenberg model, leading to the above configurations. This also applies to the HSF, $A F_{1}$ and $\mathrm{AF}_{2}$ states of the $\mathrm{V}$ tetramer. While in this case the moment differences are substantial, the basic topology of the total energy surface is unchanged. The reason for the occurrence of AS trimer state is a different one: The state is the lowest magnetic configuration compatible with the AS symmetry. Its rather high energy is explained by the artificial quenching of the center atom moment. The physical origin of the low spin states is connected with the strength of the hybridization with the substrate or the other adatoms. From the bulk we know that low spin states occur in a narrow parameter region and indicate a magnetic instability, e.g. a transition from high spin to low spin to paramagnetic states. Thus apparently for the trimers and tetramers the hybridization is sufficiently strong for these states to exist, even if they are not energetically preferred. This is in line with the observation, that the LS state does not exist for the single adatoms.

The metamagnetic behaviour of supported metallic clusters is connected with similar findings for bulk systems and clusters. The concept of different magnetic states (HSF, LSF, AF and nonmagnetic) for a given system is used to discuss properties of bulk systems like the $\alpha(\mathrm{bcc}) \rightarrow \gamma(\mathrm{fcc})$ phase transition in Fe [14] and the Invar-effect [15]. Different magnetic states in bulk systems are investigated experimentally and theoretically in a series of papers. [16-18] The possibility of more than one magnetic state in $\mathrm{V}_{9}$ and $\mathrm{Cr}_{9}$ clusters has also been explored by the calculations of Lee and Callaway. [13] They found that for some atomic spacings as many as five magnetic states exist. Thus the existence of multiple magnetic states is more common in many systems than previously assumed. 
By summarizing we conclude that the occurrence of multiple magnetic states is a typical feature for small magnetic nanostructures on noble metal surfaces. The possibility to create such nanostructures in experiments can lead to controlled changes of magnetic states in nanoscale magnets. The changes between magnetic moments of different states are proved to be large enough for an experimental observation of metamagnetic transitions in nanostructures. Magnetic fluctuations for $\mathrm{V}$ and $\mathrm{Mn}$ clusters are possible due to very small energy differences between magnetic states. Acknowledgements-We thank S. Blügel, J. Kirschner, B.K. Rao and P. Jena for helpful discussions. Financial support of the Human Capital and Mobility Program 'Ab initio (from electronic structure) calculation of complex processes in materials' of the European Union is also acknowledged. The computations are performed partially on Cray computers of the Forschungszentrum Jülich and the German supercomputer center (HLRZ).

\section{REFERENCES}

1. Eigler, D.M. and Schweizer, E.K., Nature 344, $1990,524$.

2. Brune, H., Röder, H., Borango, C. and Kern, K., Phys. Rev. Lett. 73, 1994, 1955; Röder, H., Hahn, E., Brune, H., Bucher, J.-P. and Kern, K., Nature (London) 366, 1993, 141.

3. Davies, A., Stroscio, J.A., Pierce, D.T. and Celotta, R.J., Phys. Rev. Lett. 76, 1996, 4175.

4. Gatteschi, D., Caneschi, A., Pardi, L. and Sessoli, R. Science 265, 1994, 1054.

5. Sessoli, R., Gatteschi, D., Caneschi, A. and Novak, M. A., Nature (London) 365, 1993, 141; Friedman, J. R., Sarachik, M.P., Tejada, J., Ziolo, R., Phys. Rev. Lett. 76, 1996, 3830.

6. Wildberger, K., Stepanyuk, V.S., Lang, P., Zeller, R. and Dederichs, P.H., Phys. Rev. Lett. 75, 1995, 509; Stepanyuk, V.S., Hergert, W., Wildberger, K., Zeller, R. and Dederichs, P.H., Phys. Rev. B 53, 1996, 2121.
7. Beckmann, H., Schäfer, R., Wenqi Li and Bergmann, G., Europhys. Lett. 33, 1996, 563; Schäfer, R. and Bergmann, G., Solid State Commun. 98, 1996, 45.

8. Chudnovsky, E.M. and Gunther, L., Phys. Rev. Lett. 60, 1988, 661; Chudnovsky, E.M. and Gunther, E.M., Phys. Rev. B 37, 1988, 9455.

9. Stepanyuk, V.S., Hergert, W., Wildberger, K., Zeller, R. and Dederichs, P.H. to be published.

10. Zeller, R., Lang, P., Drittler, B. and Dederichs, P.H., Mater. Res. Soc. Symp. Proc. 253, 1992, 357; Lang, P., Stepanyuk, V.S., Wildberger, K., Zeller, R. and Dederichs, P.H., Solid State Commun. 92, 1994, 755 .

11. Vosko, S.H., Wilk, L. and Nusair, M., J. Can. Phys. 58, 1200 Liu, F., Khanna, S.N. and Jena, P., Phys. Rev. B 43, $1991,8179$.

12. Douglass, D.C., Bucher, J.-P. and Bloomfield, L.A., Phys. Rev. B 45, 1992, 6341.

13. Keeyung Lee and Callaway, J., Phys. Rev. B 48, 1993, 15358; Keeyung Lee and Callaway, J., Phys. Rev. B 49, 1993, 13906; Liu, F., Khanna, S.N., and Jena, P., Phys. Rev. B 43, 1991, 8179.

14. Tauer, K.J. and Weiss, R.J., Bull. Amer. Phys. Soc. 6, 1961, 125.

15. Wassermann, E.F., J. Magn. Magn. Mater. 100 , 1991, 346.

16. Gonser, U., Krischel, K. and Nasa, S., J. Magn. Magn. Mater. 15-18, 1980, 1145.

17. Tsunoda, Y., J. Phys.: Condens. Matter 1, 1989 , 10427.

18. See, for example Kübler, J., Phys. Lett. $A$ 81, 1981, 81; Wang, C.S., Klein, B.M. and Krakauer, H., Phys. Rev. Lett. 54, 1985, 1852; Moruzzi, V.L., Marcus, P.M., Schwarz, K. and Mohn, P., Phys. Rev. B 34, 1986, 1784; Yu-mei Zhou, Nuen-qing Zhang, Lie-ping Zhong, and Ding-sheng Wang, J. Magn. Magn. Mater. 145, 1995, L237. 